\title{
Estimates of Electric Field and Its Fluctuations below a Pulsating Aurora and a Discrete Aurora
}

\author{
Takasi OGUTI and Kanji HAYASHI \\ Geophysics Research Laboratory, University of Tokyo, Tokyo, Japan
}

(Received November 22, 1984; Revised March 22, 1985)

\begin{abstract}
In order to examine a possibility for monitoring ionospheric electric fields on the ground, the fluctuation of an electric field below a pulsating aurora, as well as electric field intensity below a discrete aurora (vortex-chain aurora), or the auroral ionosphere where the electric field is supplied from the magnetospheric convection, was estimated on the basis of a simple model distribution of electric conductivity of the atmosphere, decreasing downward exponentially from the ionosphere by one order of magnitude for every $10 \mathrm{~km}$ decrease in altitude. The result shows that the fluctuation of the vertical electric field on the ground below a pulsating auroral patch could amount to about twice the horizontal electric field fluctuation in the auroral patch due to a space charge effect at and near the earth's surface. It is also shown that the vertical electric field intensity on the ground under a discrete aurora could be as large as 20 times the intensity of the horizontal electric field in the auroral ionosphere. Both the electric field and its fluctuation on the ground are shown to be much larger than those at a possible balloon altitude of $30 \mathrm{~km}$. It is suggested that the ionospheric electric field and its fluctuation could be monitored on the ground if appropriate methods such as multiple correlations between auroral luminosities in multi-domains and a ground electric field, and/or spatial correlations of ground electric fields among network stations are adopted for the data analysis of the ground electric field.
\end{abstract}

\section{Introduction}

In previous studies (OGUTI et al., 1984; OGUTI and HAYASHI, 1984), we showed that the magnetic pulsations on the ground below a pulsating aurora are fully explained by electric currents which are theoretically expected to be induced in a local enhancement of conductivity caused by a pulsating precipitation of auroral electrons. The excellent agreement between the observed and calculated magnetic field fluctuations on the ground (OGUTI and HAYASHI, 1984), indicates that the estimated electric currents must represent the real currents. This also suggests that the electric field estimated in the calculation is also a good approximation of what really takes place in and around the pulsating auroral form. This paper examines the altitude dependence of the electric field below a pulsating aurora on the basis of the electric field at the ionospheric level obtained in the previous 
paper (OGUTI and HAYASHI, 1984) in order to test the possibility of monitoring the fluctuations in the ionospheric electric field on the ground. Also the electric field intensity below a discrete (vortex-chain) aurora or below the auroral ionosphere where the convection electric field is supplied from the magnetosphere is estimated on the ground and at $30 \mathrm{~km}$ altitude, and compared with each other in relation with the measurements of electric field at a balloon altitude (e.g., MOZER and BRUSTON, 1967).

2. Fluctuations of the Electric Field in and above a Pulsating Aurora

In calculating the electric field and the electric current around an auroral patch in the previous study, the electric potential in and above the ionosphere was expressed, using ordinary polar coordinates, $r$ and $\theta$, as

$$
\begin{aligned}
& \phi^{\mathrm{o}}=\left(r \cdot E+C R^{2} / r\right) \cdot \cos \theta+\left(D R^{2} / r\right) \cdot \sin \theta \\
& \phi^{\mathrm{i}}=(E+C) \cdot r \cdot \cos \theta+D \cdot r \cdot \sin \theta .
\end{aligned}
$$

In this expression, $R$ is the radius of the local enhancement of conductivity supposed to be circular, $E$ is the ambient electric field, and $C$ and $D$ are given by

$$
\begin{gathered}
C=\frac{-\left(\left(\Sigma_{2}^{\mathrm{j}}-\Sigma_{2}^{0}\right)^{2}+\left(\Sigma_{1}^{\mathrm{i}}-\Sigma_{1}^{0}\right)\left(2 M+\Sigma_{1}^{\mathrm{i}}+\Sigma_{1}^{0}\right)\right) E}{\left(\Sigma_{2}^{\mathrm{i}}-\Sigma_{2}^{0}\right)^{2}+\left(2 M+\Sigma_{1}^{\mathrm{i}}+\Sigma_{1}^{0}\right)^{2}} \\
D=\frac{-2\left(\Sigma_{2}^{\mathrm{i}}-\Sigma_{2}^{0}\right)\left(M+\Sigma_{1}^{0}\right) E}{\left(\Sigma_{2}^{\mathrm{i}}-\Sigma_{1}^{0}\right)^{2}+\left(2 M+\Sigma_{1}^{\mathrm{i}}+\Sigma_{1}^{0}\right)^{2}}
\end{gathered}
$$

and

$$
M=\frac{\Sigma_{1}^{0}+\Sigma_{1}^{\mathrm{i}}}{2} \cdot \frac{P}{100-P}
$$

The superscripts o and $\mathrm{i}$ indicate the outside and inside of the domain, $\Sigma_{1}^{o}$ and $\Sigma_{1}^{i}$ are height-integrated Pedersen conductivities outside and inside the domain respectively, $\Sigma_{2}^{o}$ and $\Sigma_{2}^{\mathrm{i}}$ are likewise Hall conductivities, and $P$ is the fieldaligned discharge rate, defined as a percentage of the field-aligned component to the total discharge rate (field-aligned discharge plus ionospheric discharge).

These results were based on various assumptions: the ionosphere is a thin sheet, the ambient magnetic field is vertical, the conductivity enhancement is uniform in the auroral patch, the patch is circular and the field-aligned discharge current is limited to the field lines passing through the patch boundary. The agreement between observation and calculation of magnetic field fluctuations, mentioned above, supported the validity of these assumptions.

Then the electric field and the electric currents were calculated by

$$
E_{\perp}=-\operatorname{grad}_{\perp} \phi ; J_{\perp}=(\Sigma) E_{\perp} ; J_{/ /}=\operatorname{div}_{\perp} J_{\perp}
$$

using the Hall/Pedersen conductivity ratio, conductivity enhancement factor, and 
field-aligned discharge rate as parameters.

An example of the calculation of electric currents in and around the domain is reproduced from the previous paper (OGUTI and HAYASHI, 1984) in Figs. 1(a) to (d) for the convenience of readers. Figure 1(a) shows the total electric current including the ambient (jet) current, while Figure 1(b) is the additional current due to the conductivity enhancement of the domain, obtained by subtracting the uniform ambient current from the total current in Fig. 1(a). The additional current in Fig. 1(b) is divided into two parts, field-aligned pair currents connecting in the domain, and a twin-vortex current which closes in the ionosphere connecting inside to the outer part of the additional current in Fig. 1(b) without divergence at the boundary. Figure 1(c) indicates the field-aligned pair currents and Fig. 1(d) the twin-vortex current, thus separated. These two currents were shown to fully explain the magnetic deflection on the ground below a pulsating auroral patch.

Figures 2(a) and (b) illustrate the deformation of the ambient electric field and the additional electric field around the conductivity enhancement in the same calculation which are produced by the space charge at the domain boundary. The additional electric field contributes to the fluctuation of the electric field around the pulsating auroral patch. In contrast to the magnetospheric dynamo, the electromotive force here is produced by the local conductivity enhancement in the ionosphere. That is, the ionospheric current which is directly connected to the field-aligned pair currents in Fig. 1(c) is the source of the charge separation at the boundary of the domain. Therefore, the electric field is reversed to the ionospheric part of the field-aligned pair currents inside the domain, and it extends outside in the ordinary two dimensional form, as shown in Fig. 2(b). Although a similar calculation of electric current around a local enhancement of conductivity in the ionosphere was carried out by STUBBE and KoPKA (1977) in relation to ionospheric modification experiments, they neglected the field-aligned discharge at the boundary so that the field-aligned pair current is missing in their result. The calculation of Figs. 1(a) to (d) and Figs. 2(a) and (b) is based on a Hall/Pedersen conductivity ratio of 2 , a conductivity enhancement factor of the domain of 1.5 , and a field-aligned discharge rate of $55 \%$. The $55 \%$ fieldaligned discharge rate was empirically determined by comparing results of calculation with observed magnetic variations (for details, see OGUTI and HAYASHI, 1984).

An important point of the calculation that was not much discussed in our previous study is that it predicts the pattern and the intensity of the electric field which is additionally produced around the pulsating auroral patches as the auroral patch pulsates. Two examples of calculation, of the intensity and direction of the additional electric field in and above the ionosphere as function of the field-aligned discharge rate are also reproduced in Figs. 3(a) and (b). As seen in Figs. 3(a) and (b), the intensity of the additional electric field in the domain, for a field-aligned discharge rate of $55 \%$, is about $20 \%$ of the ambient (convection) electric field when the conductivity in the domain is 1.5 times larger 


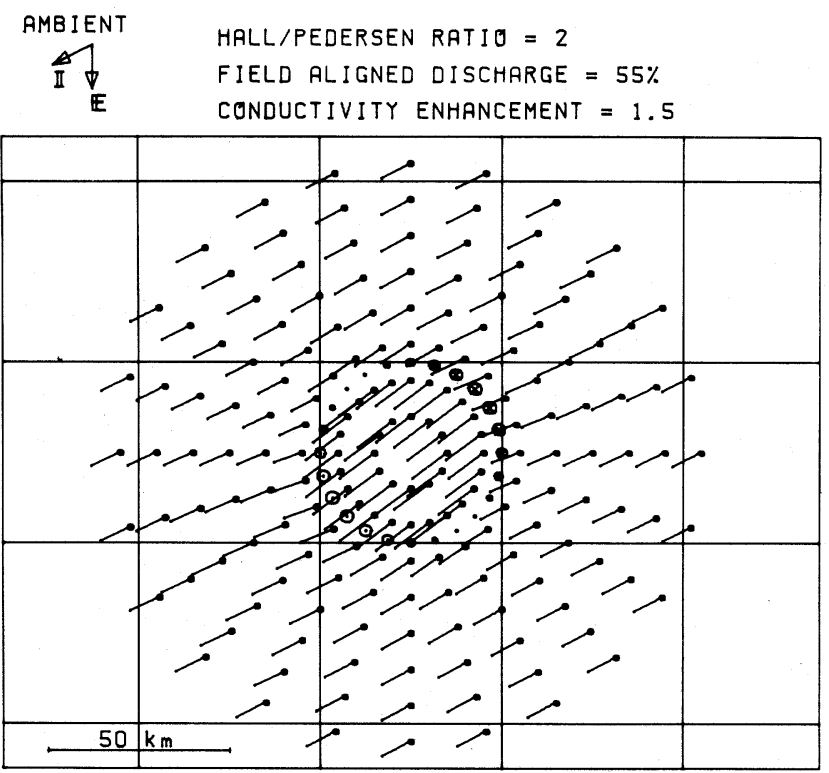

ELECTRIC CURRENT AROUND A DOMAIN

(a)
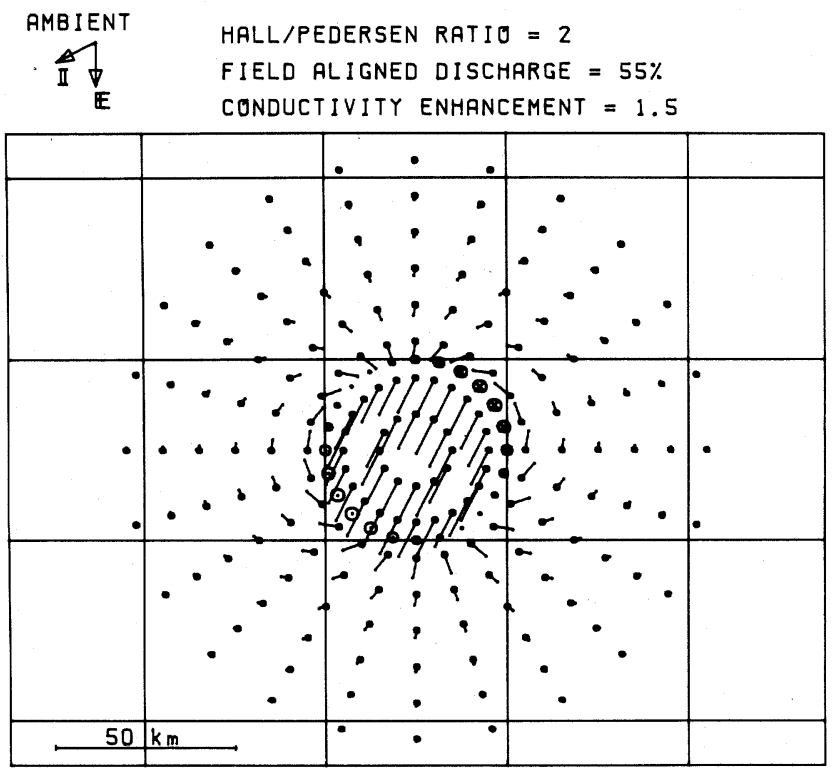

ADDITIONAL ELECTRIC CURRENT AROUND A DOMAIN

(b)

Fig. 1. An example of the electric current calculated in the vicinity of a conductivity enhancement (brightened auroral) domain. (a) The total current including the uniform, ambient current. (b) Additional electric current induced in the vicinity of the conductivity enhancement region. (c) 


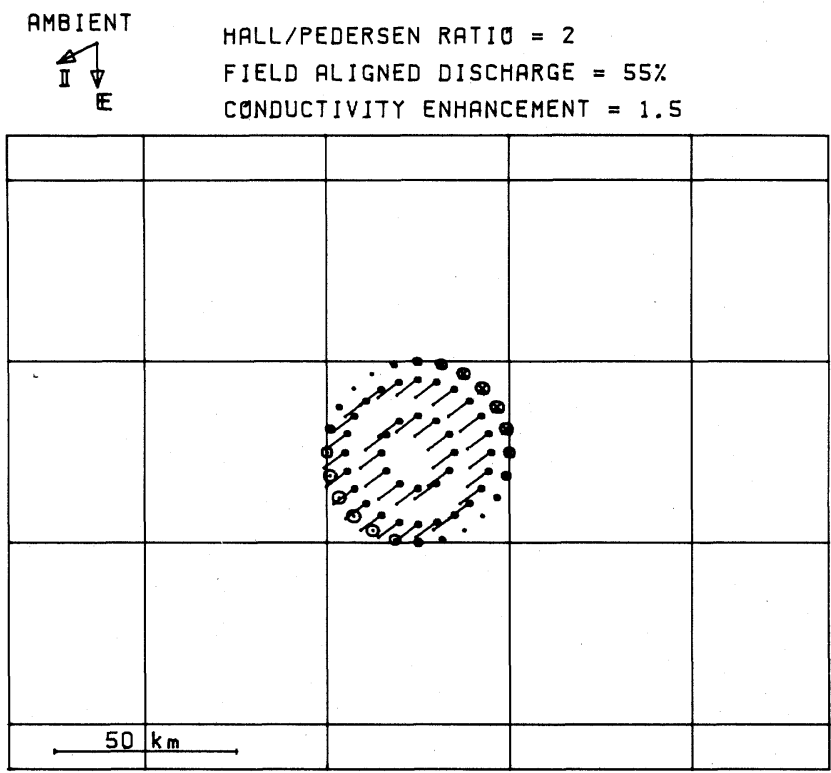

FIELD RLIGNED PAIR CURRENT AROUND A DOMAIN

(c)

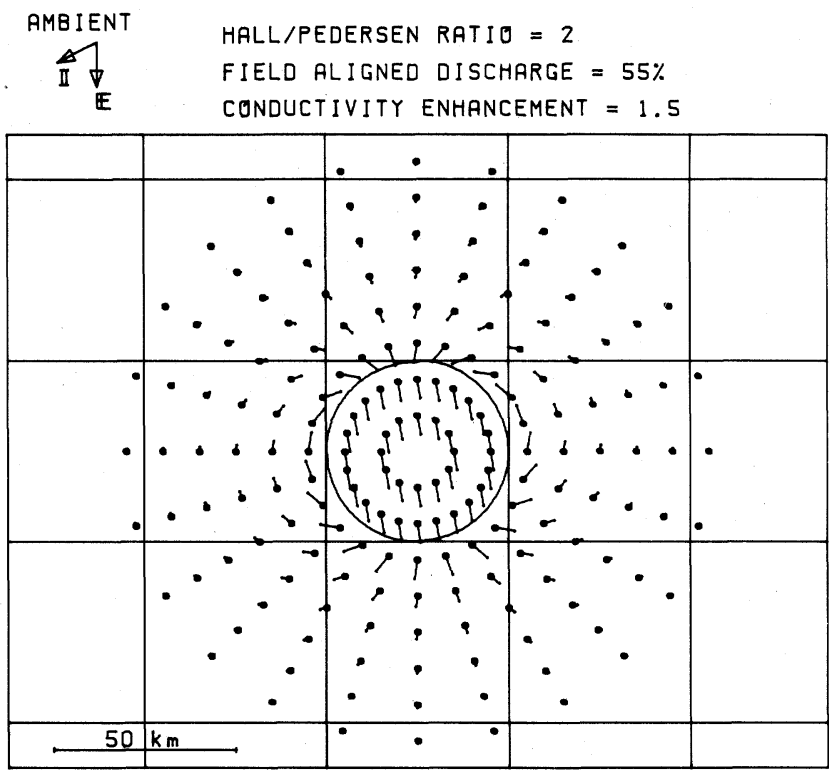

TWIN VORTEX CURRENT AROUND A DOMAIN

(d)

A field-aligned pair current, connected in the conductivity enhancement region. (d) A twinvortex current which connects inside with the outside part of the additional current in Fig. 1(b) without divergence at the boundary. 

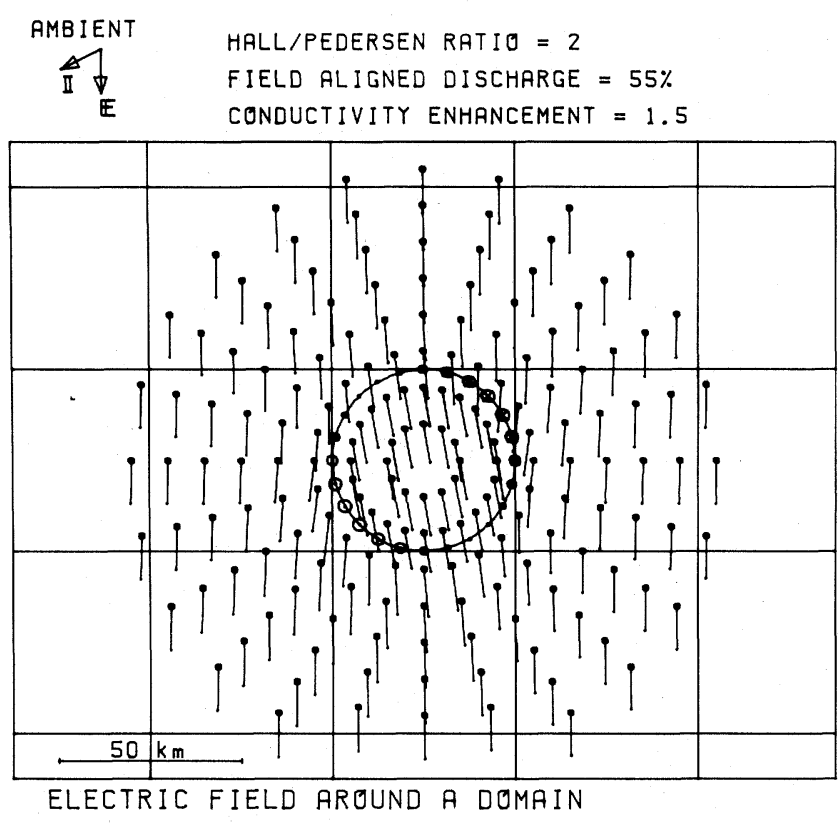

(a)

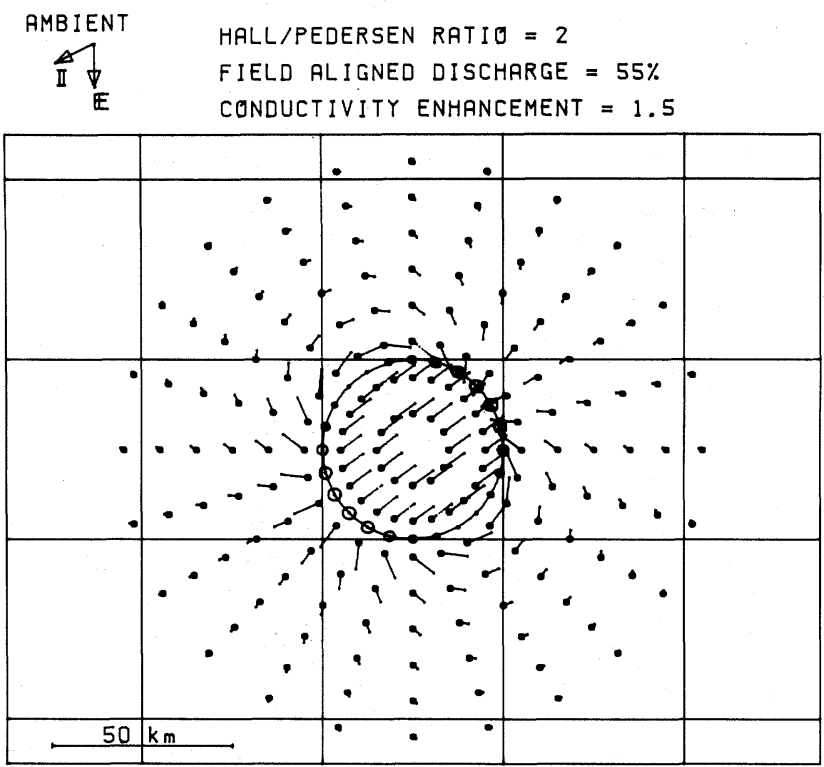

ADDITIONAL ELECTRIC FIELD RROUND A DOMAIN

(b)

Fig. 2. An example of the electric field calculated around a conductivity enhancement (brightened auroral) domain corresponding to the currents in Fig. 1. (a) Total ionospheric (and higher) electric field including the ambient field. (b) Additional electric field around the conductivity enhancement. 

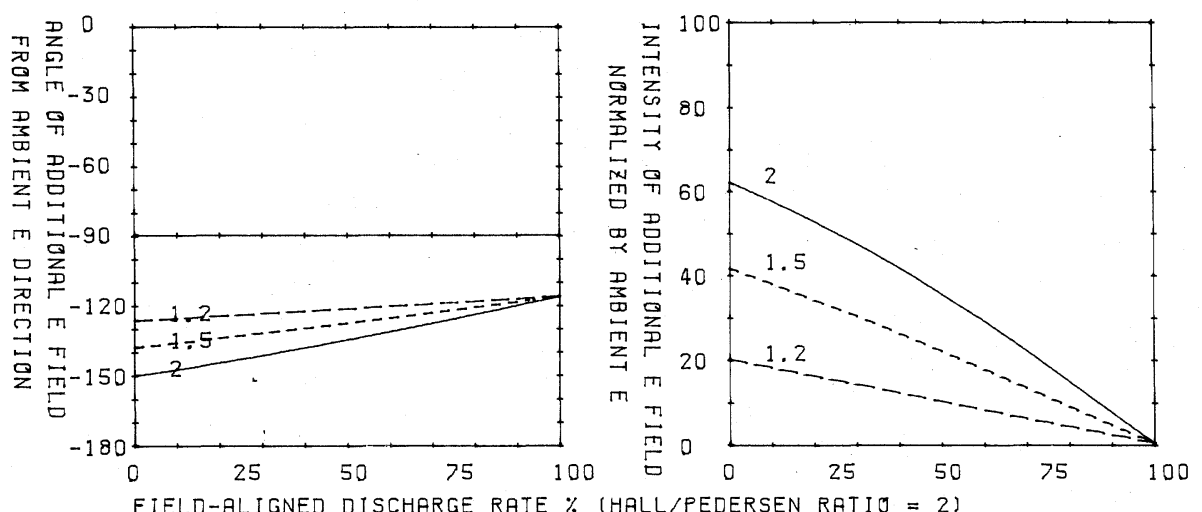

(a)
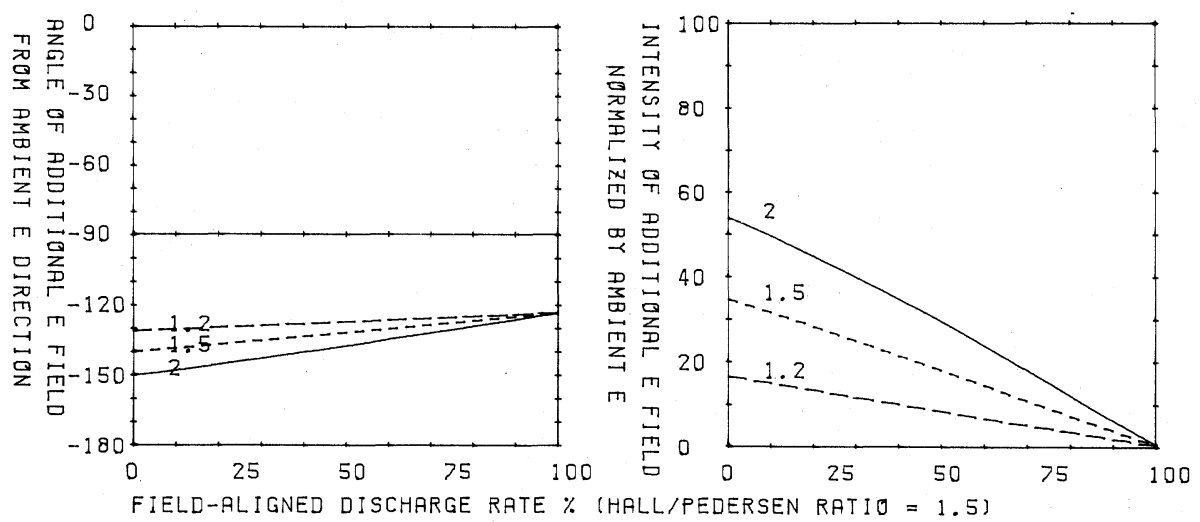

(b)

Fig. 3. The calculated changes in direction (left) and intensity (right) of the additional electric field in a domain versus the field-aligned discharge rate. The direction is given in an angle from the ambient electric field, and the intensity is normalized by the ambient field intensity. (a) Hall/Pedersen ratio of 1.5 . (b) Hall/Pedersen ratio of 2.

than that of the background and it is reduced to about $10 \%$ when the conductivity enhancement factor is 1.2 .

\section{Derivation of Fundamental Equations}

The additional electric field produced around an auroral patch penetrates downward into the atmosphere as shown by MOzER and SERLIN (1969) and by PARK (1976), and may be observed by balloon below pulsating auroras. The distribution of the electric field fluctuations below a pulsating aurora is estimated 
using Fourier-Bessel expansions of the ionospheric electric field in Fig. 2(b).

Here, the conductivity in the atmosphere below the ionosphere is assumed to be isotropic and decreasing exponentially as $\exp (-\alpha z)$ (downward positive for $z$ ) with a decrease in altitude. Both assumptions are shown to be reasonable enough to estimate gross feature of the electric field below the ionosphere as seen in some of previous studies (e.g., PARK, 1976). A stationary limit is dealt with here for simplicity. This is valid for variations with a period longer than 10 seconds (MiURA et al., 1982). Hence, the electric potential below the ionosphere, $\phi_{\mathbf{a}}$, satisfies the relation as

$$
\partial^{2} \phi_{\mathrm{a}} / \partial x^{2}+\partial^{2} \phi_{\mathrm{a}} / \partial y^{2}+\partial^{2} \phi_{\mathrm{a}} / \partial z^{2}-\alpha \partial \phi_{\mathrm{a}} / \partial z=0
$$

due to the source-free condition of the current, $\operatorname{div} I=0$. The solution of this equation is, using ordinary cylindrical coordinates, $r, \theta$ and $z$, given as

$$
\begin{aligned}
\phi_{\mathrm{a}}= & \sum_{k n} J_{n}(k r) \sin (n \theta)\left(A_{n}^{k} \exp \left[\left\{\alpha-\left(\alpha^{2}+4 k^{2}\right)^{1 / 2}\right\} z / 2\right]\right. \\
& \left.+B_{n}^{k} \exp \left[\left\{\alpha+\left(\alpha^{2}+4 k^{2}\right)^{1 / 2}\right\} z / 2\right]\right) \\
& +\sum_{k n} \sum_{n} J_{n}(k r) \cos (n \theta)\left(C_{n}^{k} \exp \left[\left\{\alpha-\left(\alpha^{2}+4 k^{2}\right)^{1 / 2}\right\} z / 2\right]\right. \\
& \left.+D_{n}^{k} \exp \left[\left\{\alpha+\left(\alpha^{2}+4 k^{2}\right)^{1 / 2}\right\} z / 2\right]\right)
\end{aligned}
$$

where the coefficients $A_{n}^{k}, B_{n}^{k}, C_{n}^{k}$ and $D_{n}^{k}$ are determined by the ionospheric and ground boundary conditions.

As seen in Fig. 2(b), the model electric field in the ionosphere here is given by a single azimuthal wave number, $n=1$, at $z=0$. Therefore, using a coordinate system along the additional electric field direction in the domain, Equation (7) is simplified as

$$
\begin{aligned}
\phi_{\mathrm{a}}= & \sum_{k} J_{1}(k r) \sin (\theta)\left(A_{1}^{k} \exp \left[\left\{\alpha-\left(\alpha^{2}+4 k^{2}\right)^{1 / 2}\right\} z / 2\right]\right. \\
& \left.+B_{1}^{k} \exp \left[\left\{\alpha+\left(\alpha^{2}+4 k^{2}\right)^{1 / 2}\right\} z / 2\right]\right) .
\end{aligned}
$$

On the other hand, assuming uniform, finite ground conductivity, the electric potential below the ground surface, $\phi_{\mathrm{g}}$, is given by

$$
\phi_{\mathrm{g}}=\sum_{k} J_{1}(k r) \sin (\theta) E_{1}^{k} \exp (-k z)
$$

Eliminating $E_{1}^{k}$ by use of Eqs. (8) and (9) and two boundary conditions at the earth's surface $\left(z=z_{0}\right)$, that is, the continuity of the normal electric current and tangential electric field, we obtain a relation between $B_{1}^{k}$ and $A_{1}^{k}$ as

$$
B_{1}^{k}=-A_{1}^{k} \exp \left\{-\left(\alpha^{2}+4 k^{2}\right)^{1 / 2} z_{0}\right\} \frac{1+\left(\sigma_{\mathrm{U}} / \sigma_{\mathrm{L}}\right)\left\{\alpha-\left(\alpha^{2}+4 k^{2}\right)^{1 / 2}\right\} / 2 k}{1+\left(\sigma_{\mathrm{U}} / \sigma_{\mathrm{L}}\right)\left\{\alpha+\left(\alpha^{2}+4 k^{2}\right)^{1 / 2}\right\} / 2 k}
$$


where $\sigma_{\mathrm{U}}$ and $\sigma_{\mathrm{L}}$ denote electric conductivities of the atmosphere on the surface and of the ground, respectively. This equation is simplified, when the ground conductivity, $\sigma_{\mathrm{L}}$, is large enough for neglecting the second term in the denominator, as

$$
B_{1}^{k}=-A_{1}^{k} \exp \left\{-\left(\alpha^{2}+4 k^{2}\right)^{1 / 2} z_{0}\right\}
$$

in a limit of an infinite ground conductivity.

The criterion for this is that the conductivity ratio, $\sigma_{\mathrm{U}} / \sigma_{\mathrm{L}}$, is much smaller than $2 k /\left[\alpha+\left(\alpha^{2}+4 k^{2}\right)^{1 / 2}\right]$. Since the ratio $k$, the wave number of the ionospheric electric field, to $\alpha$, the wave number of the conductivity distribution in the atmosphere, here in this calculation is $10^{-2}$ at least, the conductivity ratio, $\sigma_{\mathrm{U}} / \sigma_{\mathrm{L}}$, when smaller than $10^{-4}$, guarantees the validity of Eq. (11) within $1 \%$ accuracy. The conductivity ratio is usually smaller, and hence, the equipotential assumption of the earth's surface is found to be a good approximation for estimating electric field distributions above the earth's surface.

Equation (8) is then rewritten as

$$
\begin{aligned}
\phi_{\mathrm{a}}= & \sum_{k} A_{1}^{k} J_{1}(k r) \sin \theta\left(\exp \left[\left\{\alpha-\left(\alpha^{2}+4 k^{2}\right)^{1 / 2}\right\} z / 2\right]\right. \\
& \left.-\exp \left[-\left(\alpha^{2}+4 k^{2}\right)^{1 / 2} z_{0}+\left\{\left(\alpha^{2}+4 k^{2}\right)^{1 / 2}+\alpha\right\} z / 2\right]\right) .
\end{aligned}
$$

Considering an area large enough to justify the neglect of the electric field contribution from the outside, the coefficients $A_{1}^{k}$ are determined by expanding the ionospheric potential distribution at $z=0$, given by Eq. (1), into a Fourier"Bessel series as

$$
\begin{aligned}
A_{1}^{k} s\left[1-\exp \left\{-\left(\alpha^{2}+4 k^{2}\right)^{1 / 2} z_{0}\right\}\right] & =\frac{2 G}{\left[J_{2}\left(k_{s}\right)\right]^{2} k_{s}}\left[\int_{0}^{a} r^{2} J_{1}\left(k_{s} r\right) \mathrm{d} r / a+\int_{a}^{1} a J_{1}\left(k_{s} r\right) \mathrm{d} r\right] \\
& =\frac{2 G_{a}}{\left[J_{2}\left(k_{s}\right)\right]^{2} k_{s}}\left[J_{2}\left(k_{s} a\right)-J_{0}\left(k_{s}\right)+J_{0}\left(k_{s} a\right)\right]
\end{aligned}
$$

where $k_{s}$ is the $s$-th root of $J_{1}, a$ is the radius of the auroral domain normalized by the radius of the whole area under consideration and $G$ is a constant to be determined by the potential at the ionospheric boundary $(z=0)$. The first and the second integrations come from $\phi^{\mathrm{i}}$ and $\phi^{\circ}$ of Eq. (1), respectively. Then, with $k$ replaced by $k_{s}$, the electric potential below the ionosphere is eventually expressed by Eq. (12) taking the summation by $s$ with $A_{1}^{k} s$ determined by Eq. (13).

\section{Estimates of Electric Field Fluctuations below a Pulsating Aurora}

On the basis of the potential distribution in the atmosphere derived in the previous section, we will discuss a possible feature of electric field fluctuations below a pulsating aurora. 


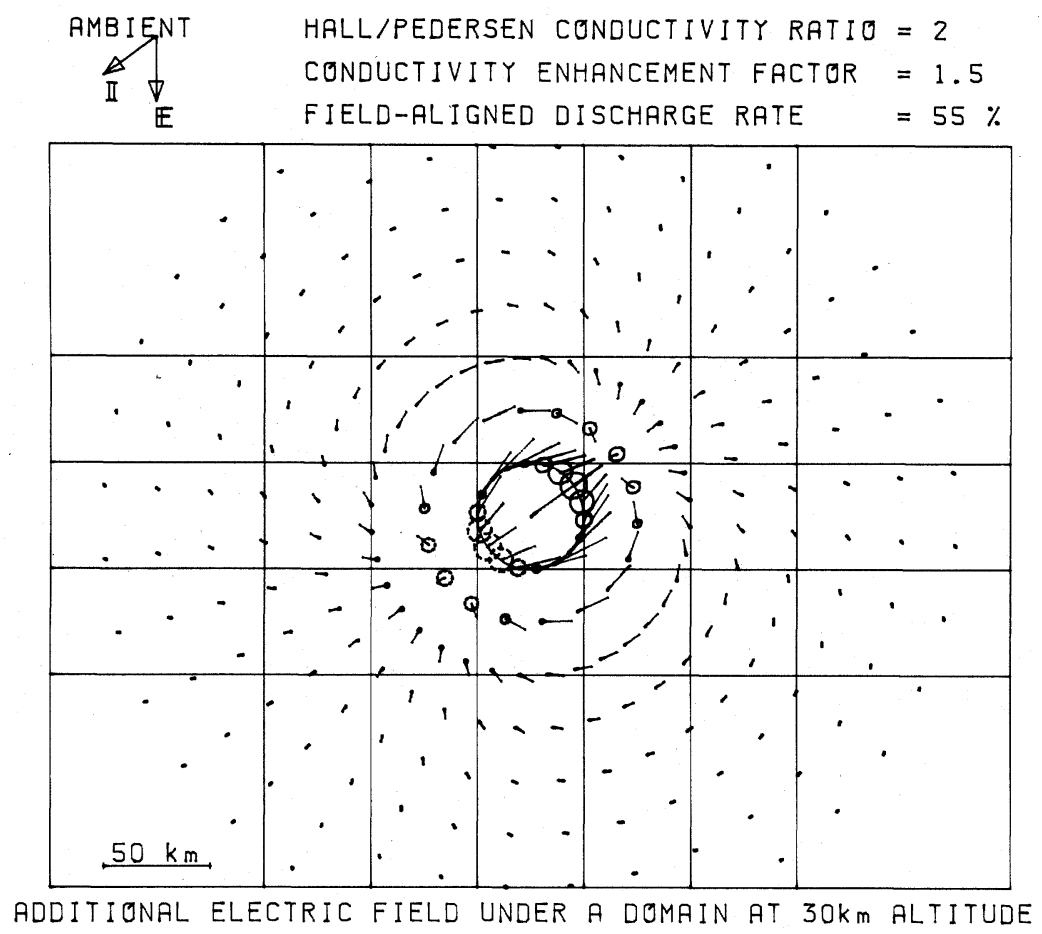

(a)

First we would like to note that the attenuation of the ionospheric part of the electric potential with distance, $z$, is much reduced owing to a steep decrease in electric conductivity below the ionosphere. As seen in Eqs. (7), (8) and (12), the first term, that is the electric potential penetrating downward from the ionosphere, would decrease as $\exp (-k z)$ with a decrease in altitude in the vacuum or in a uniform conductivity region, where $\alpha=0$, while the decreasing rate in the atmosphere with a distribution of conductivity, $\exp (-\alpha z)$, is reduced to $\exp \left[\left\{\alpha-\left(\alpha^{2}+4 k^{2}\right)^{1 / 2}\right\} z / 2\right]$. Corresponding to this, the second part of the electric potential (the second term) is induced by the space charge at the earth's surface.

The downward penetration of the horizontal component of the electric field is exactly the same as that of the potential. As seen in the form of ex$\mathrm{p}\left[\left\{\alpha-\left(\alpha^{2}+4 k^{2}\right)^{1 / 2}\right\} z / 2\right]$, the downward penetration is determined by the horizontal wave number, $k$, and the conductivity change, $\alpha$. As $k$ decreases, or as $\alpha$ increases, the penetration becomes deeper. It is also known in this expression that the effect of a change in $\alpha$ is larger for larger $k$. This indicates that the reduction of the decreasing rate due to steep decrease in conductivity is more effective for the electric field with a smaller horizontal extent. The behavior of the vertical component, however, is a little bit different from this. The downward penetration of the vertical component, with the same form as the potential and 


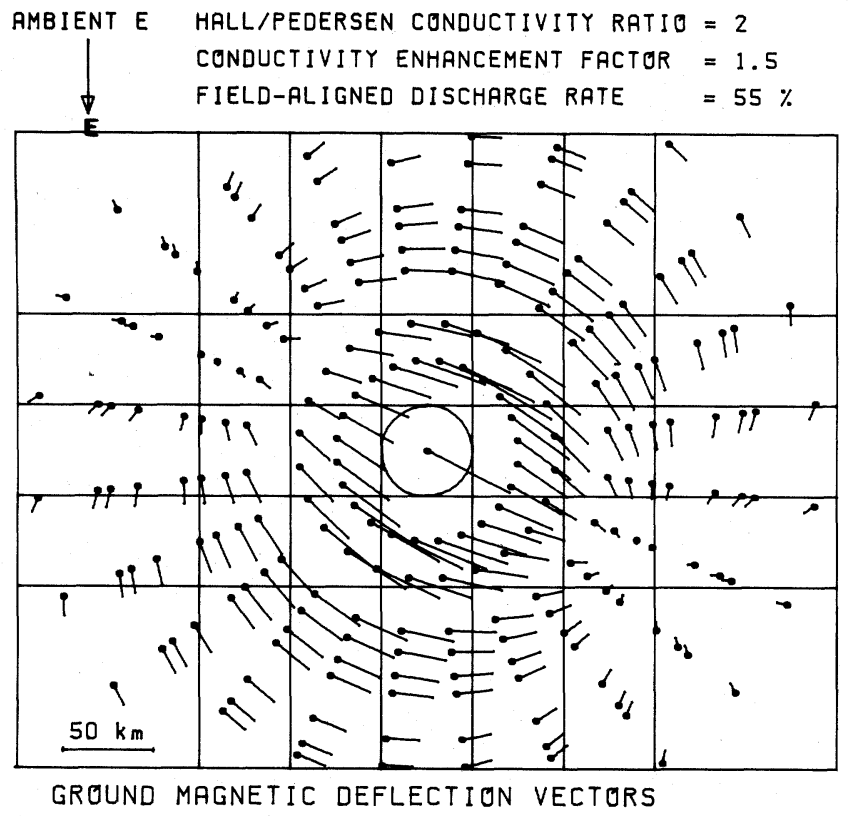

(b)

Fig. 4. (a) Distribution of the fluctuation electric field at a $30 \mathrm{~km}$ altitude. Vertical intensities are given by circles (open upward, dashed downward) and horizontal components are given by arrows from the centers of circles. Note that both the divergence and the convergence are about $40 \mathrm{~km}$ apart from the center of the conductivity enhancement (brightened auroral) domain. (b) Distribution of horizontal magnetic deflection vectors on the ground below a conductivity enhancement domain (auroral patch). A pair of convergence and divergence of vectors appears at about $120 \mathrm{~km}$ from the center.

the horizontal component but multiplied by a factor $\left\{\alpha-\left(\alpha^{2}+4 k^{2}\right)^{1 / 2}\right\} / 2$, generally depends on the height of the ionosphere, $z_{0}$, in addition on $k$ and $\alpha$. As $\alpha$ varies from 0 to $\infty,\left\{\left(\alpha^{2}+4 k^{2}\right)^{1 / 2}-\alpha\right\} / 2$ does from $k$ to 0 . Therefore, the critical level, $z_{\mathrm{c}}$, where the intensity of the downward penetrating electric field changes the sign of its $\alpha$-dependence, and which is given as $z_{\mathrm{c}}=2 /\left\{\left(\alpha^{2}+4 k^{2}\right)^{1 / 2}-\alpha\right\}$, changes from $1 / k$ to $\infty$. The downward penetrating vertical electric field increases with an increase in $\alpha$ at $z>z_{\mathrm{c}}$ whereas it decreases at $z<z_{\mathrm{c}}$. Since $1 / k$, the horizontal wave length of the electric field, is broadly distributed from 0 to some hundreds $\mathrm{km}$, the earth's surface $\left(z=z_{0}\right)$ is located at $z>z_{\mathrm{c}}$ for an electric field with a small horizontal extent $\left(1 / k<z_{0}\right)$, while the situation is reversed as $z<z_{\mathrm{c}}$ for that with a large horizontal extent $\left(1 / k>z_{0}\right)$. Consequently, the downward penetrating vertical electric field increases with increase in $\alpha$ when the characteristic horizontal extent of the electric field is smaller than $z_{0}$, while it decreases when the extent is larger than $z_{0}$. This, together with the fact that the decreasing rate 
of the horizontal component is reduced to $\exp \left[\left\{\alpha-\left(\alpha^{2}+4 k^{2}\right)^{1 / 2}\right\} z / 2\right]$, is an essential factor which allows the electric field with a small horizontal extent, such as is due to a pulsating aurora, to penetrate well down to the lower altitudes.

An estimate of the electric field fluctuation below a pulsating auroral domain is shown in Figs. 4 and 5. Figure represents the electric field distribution at a possible balloon altitude of $30 \mathrm{~km}$ along with the magnetic deflections on the ground, reproduced from the previous study (OGUTI and HAYASHI, 1984), and Figure 5 shows distributions of the electric field fluctuation in a vertical section, which includes the symmetry axis of the additional electric field, both on the basis of the parameters, the Hall/Pedersen conductivity ratio of 2 , the conductivity enhancement factor of 1.5 and field aligned discharge rate of $55 \%$. In calculation, it is assumed that the atmospheric conductivity below the ionosphere decreases one order of magnitude with every $10 \mathrm{~km}$ decrease in altitude.

Note that the divergence and the convergence in Fig. 4(a) are only about $40 \mathrm{~km}$ apart from the center of the conductivity enhancement domain (brightened auroral patch) in contrast to the $120 \mathrm{~km}$ distance of those of the ground magnetic deflection pattern in Fig. 4(b). It is easy to show that the difference is due essentially to the conductivity distribution below the ionosphere as mentioned above, and not to the difference in the observation altitudes, the balloon altitude for the electric field (Fig. 4(a)) or the earth's surface for the magnetic field (Fig. 4(b)). This is known in Fig. 5 because the electric field distribution in the ionosphere appears to penetrate almost vertically downward below the ionosphere.

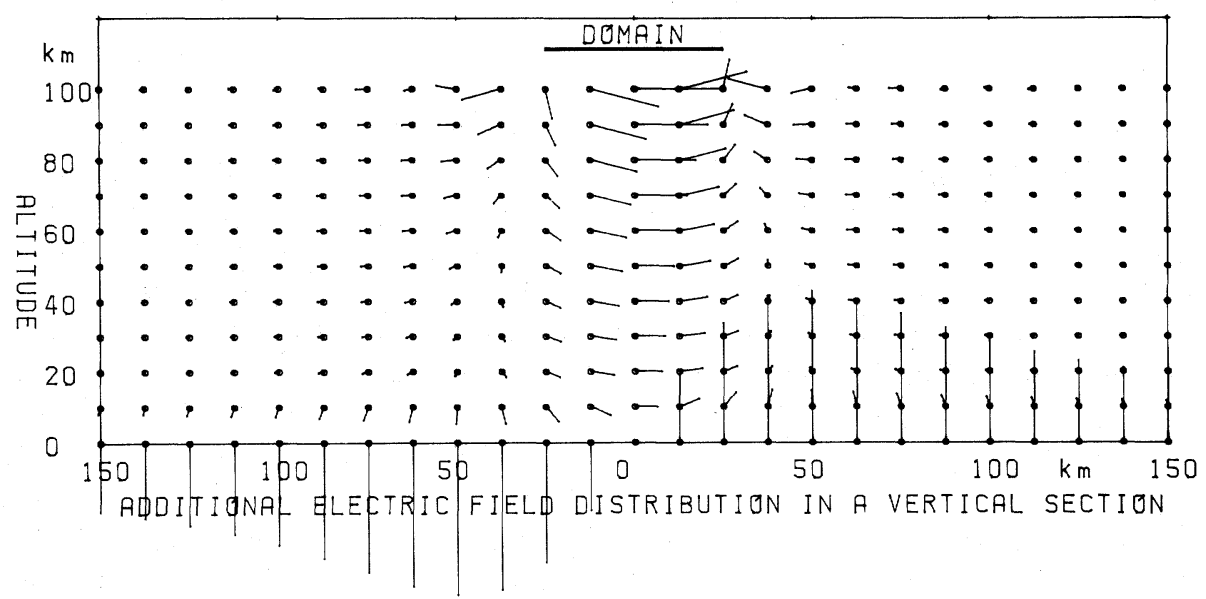

Fig. 5. Distribution of the estimated fluctuation electric field below a conductivity enhancement (brightened auroral) domain in a vertical section including symmetry axis of the additional electric field. 
It is seen in Fig. 5 that the fluctuation intensity at a $30 \mathrm{~km}$ altitude under a pulsating domain is approximately $35 \%$ of the additional electric field in the domain. This, together with the results in Figs. 3(a) and (b), indicates that the fluctuation in electric field intensity related to a pulsating auroral patch, when measured directly under the patch, is about $7 \%$ of the ambient convection electric field in the case of 1.5 times conductivity enhancement. This reduces to $3.5 \%$ in the case of 1.2 times enhancement.

Although we used a $50 \mathrm{~km}$ scale size of the patch in the calculation corresponding to the $50 \mathrm{~km}$ grid size in the analysis in the previous study (OGUTI and HAYASHI, 1984), actual patch size is usually smaller so that the field also tends to be smaller. Hence, the measurement of the fluctuation electric field below a pulsating aurora by a balloon would be quite difficult because of the contamination of atmospheric components. The fluctuation electric field may be detectable at the balloon altitude only when a patch is much larger than the domain size here, $50 \mathrm{~km}$ across.

As seen in the discussion above, the pattern of the electric field fluctuation is predictable so that the pattern may serve for distinguishing the ionospheric component out of the measured electric field. In order to separate the ionospheric component, however, the multiple correlation analysis between electric field fluctuations and auroral luminosities in multi-domains, will again be required because the electric field here, in the same way as magnetic deflections on the ground in the previous study, is the spatial integration of the contributions from various patches.

A steep increase in the vertical electric field in the vicinity of the earth's surface is another important feature of the electric field in the atmosphere with a gradient of electric conductivity. This is equivalent to the fact that a high potential difference occurs at a high resistivity in a circuit net consisting of various resistors. As seen in Eq. (12), the vertical electric field intensity is reduced to one half with a $3-3.5 \mathrm{~km}$ increase in altitude above the surface. Equation (12) also shows that the electric field is not enhanced at the earth's surface if the atmospheric conductivity is uniform or in a vacuum environment where $\alpha=0$. The effect of the enhancement on the ground is seen in Fig. 5. The vertical component of the electric field fluctuation at the ground surface is about 4 times larger than the horizontal component at the balloon altitude, and amounts to about twice that in the auroral patch. Since the electric field fluctuation in an auroral patch amounts to $20 \%$ as large as the ambient field, this means that the electric field fluctuation on the ground could be about a half the intensity of the ambient electric field in the ionosphere.

The intensity of the additional electric field below the ionosphere highly depends on the horizontal size of the auroral patch. An estimation of the scale dependence of the vertical field intensity on the ground is shown in Fig. 6. It is known in this figure that the vertical field intensity rapidly decreases with a decrease in the horizontal size, $L$, of the patch, from a value of about twice that in a patch with a size of $50 \mathrm{~km}$ across to a few percent of that in a 10 


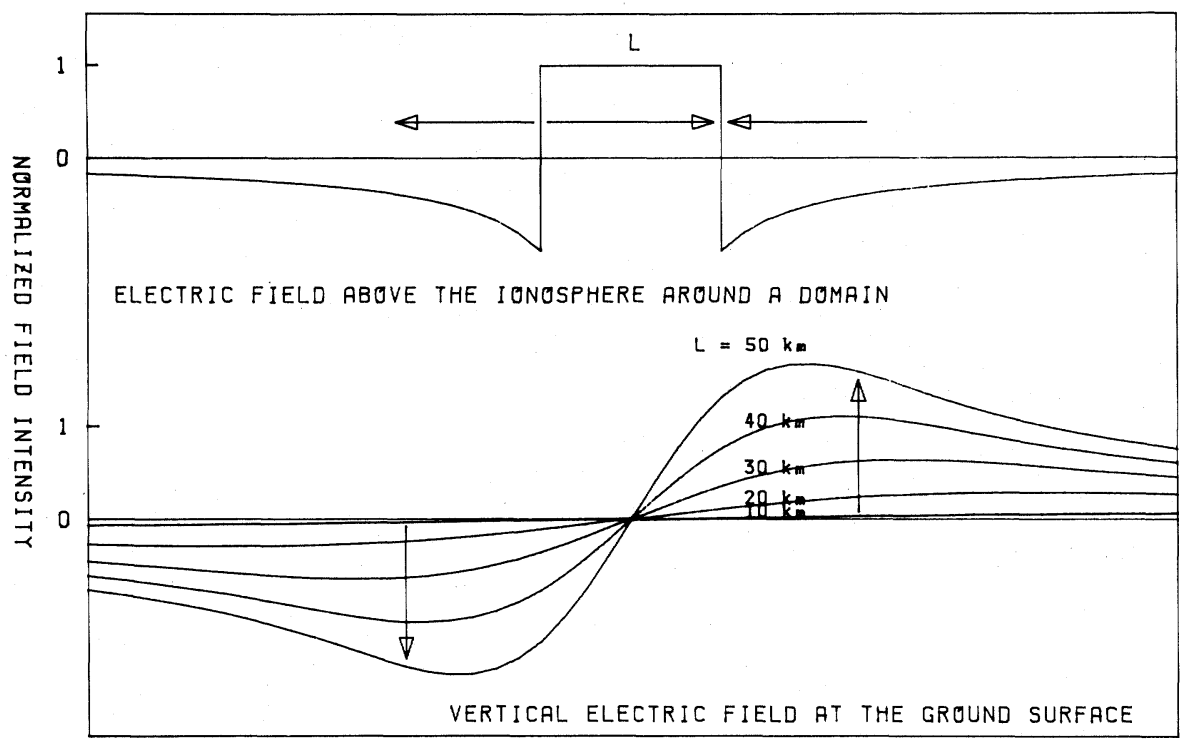

Fig. 6. Spatial distribution of the vertical electric field intensity expected on the ground below a conductivity enhancement domain (brightened auroral patch) of a pulsating aurora. The ground electric field intensity is found to depend highly on the size of the auroral patch, $L$.

$\mathrm{km}$ size patch. The vertical electric field fluctuation on the ground caused by *a pulsating auroral patch may thus be measurable only when the patch size is larger than several tens of $\mathrm{km}$.

5. Electric Field Associated with a Discrete Auroral Form or the Convection Electric Field

The consideration above can also be applied to a discrete aurora, which is regarded to be a vortex chain (OGUTI, 1981), or to the ionospheric electric field caused by the magnetospheric convection. Since the vortex-chain nature of a discrete aurora indicates a temporal enhancement of electric field convergent towards the auroral arc, elongated in the east-west direction, the electric field at the ionospheric level here is assumed to be a two-dimensionally convergent field with a uniform intensity. Equation (7) is then modified to a simpler form as

$$
\begin{aligned}
\phi_{\mathrm{a}}= & \sum_{n} \sin (n x)\left(A n \exp \left[\left\{\alpha-\left(\alpha^{2}+4 n^{2}\right)^{1 / 2}\right\} z / 2\right]+B_{n} \exp \left[\left\{\alpha+\left(\alpha^{2}+4 n^{2}\right)^{1 / 2}\right\} z / 2\right]\right) \\
& +\sum_{n} \cos (n x)\left(C_{n} \exp \left[\left\{\alpha-\left(\alpha^{2}+4 n^{2}\right)^{1 / 2}\right\} z / 2\right]+D_{n} \exp \left[\left\{\alpha+\left(\alpha^{2}+4 n^{2}\right)^{1 / 2}\right\} z / 2\right]\right)
\end{aligned}
$$


where $x$ is the distance from the center of the convergent electric field in the north-south direction. Here again using the equipotential condition on the ground, this equation is rewritten as

$$
\begin{aligned}
\phi_{\mathrm{a}}= & \sum_{n}\left[A_{n} \sin (n x)+C_{n} \cos (n x)\right]\left(\operatorname { e x p } \left[\left\{\alpha-\left(\alpha^{2}+4 n^{2}\right)^{1 / 2}\right\} z / 2\right.\right. \\
& \left.-\exp \left[-\left(\alpha^{2}+4 n^{2}\right)^{1 / 2} z_{0}+\left\{\alpha+\left(\alpha^{2}+4 n^{2}\right)^{1 / 2}\right\} z / 2\right]\right)
\end{aligned}
$$

and the constants $A_{n}$ and $C_{n}$ are determined expanding a given ionospheric potential distribution into a Fourier series.

Assuming again that the electric conductivity below the ionosphere decreases one order of magnitude for every $10 \mathrm{~km}$ decrease in altitude, the electric field distribution in the meridian plane is estimated as shown in Fig. 7(a). Here, it is assumed that a two-dimensionally convergent electric field in the north-south direction exists above the ionosphere within an east-west elongated belt $200 \mathrm{~km}$ in width, with a uniform intensity. The electric field intensity at $30 \mathrm{~km}$ altitude is roughly the same as the horizontal field intensity in the ionosphere as seen in the figure. On the other hand, the vertical electric field on the ground under the convergence region in the ionosphere is found to be about 20 times larger than the ionospheric electric field. The change in the ground electric field intensity with change in the characteristic width, $L$, is shown in Fig. 7(b). Again, a strong dependence of the ground field intensity on the scale is evident. It is about 20 times when the width is $200 \mathrm{~km}$, and decreases to roughly the same value as in the ionosphere when the size is reduced to $40 \mathrm{~km}$.

* These results indicate that the horizontal electric field in the ionosphere with an intensity of $50 \mathrm{mV} / \mathrm{m}$ causes the vertical electric field on the ground with an intensity of up to $1 \mathrm{~V} / \mathrm{m}$ when the ionospheric electric field is distributed in a belt of $200 \mathrm{~km}$ in width. Then, the contribution of the ionospheric electric field to the vertical electric field on the ground is estimated to be about $1 \%$ of the background atmospheric electric field near the surface, which usually amounts to $100 \mathrm{~V} / \mathrm{m}$ on an average.

The $1 \%$ contribution of the vertical electric field suggests that it is fairly difficult to distinguish the ionospheric component from the background, if it is stationary, because of a possible variability of the background. However, the result also shows how the distribution of the ionospheric contribution should be in relation to the auroral distribution or the configuration of the ionospheric electric field. If the ionospheric electric field intensity increases the resultant variation in the vertical electric field on the ground must be distributed as shown in Fig. 7. On the other hand, if the auroral belt moves in the north-south direction, the electric field variations on the ground should be accounted for in terms of spatial differentiation of the distribution pattern in Fig. 7. The temporal variations of the vertical electric field synchronous to an auroral brightening or movement must be distributed systematically as expected from the distribution of the calculated electric field. Therefore, if the vertical electric field components vary- 


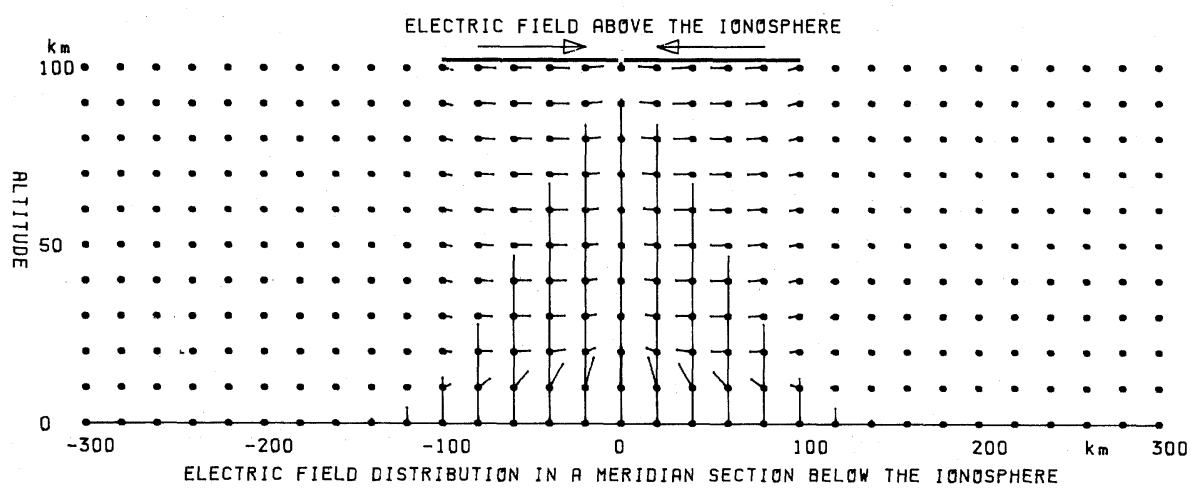

(a)

ing synchronously with auroral activity could be separated from the background, and if its spatial distribution could be determined by network observations, the ionospheric electric field distribution could be estimated by comparing it with expected variations. Spatial correlations of the variations at network stations would be useful for distinguishing the ionospheric contribution from the vertical electric field on the ground, taking the possible distributions into consideration.

\section{Conclusion and Discussion}

The possible distribution pattern and the intensity of electric field fluctuation below a pulsating aurora, and a discrete aurora, or the auroral ionosphere with the convection electric field, were examined in this paper. Conclusions are summarized as follows;

1) The intensity of the electric field fluctuation due to a pulsating auroral patch that is $50 \mathrm{~km}$ across will be a few percent of the ambient convection electric field, with a pair of divergence and convergence of the horizontal field component, both about $40 \mathrm{~km}$ apart from the center of the brightened domain at a $30 \mathrm{~km}$ altitude. The contribution of a pulsating patch to the vertical electric field on the ground is approximately twice the additional electric field in the brightened auroral patch and about half the convection electric field.

2) The intensity of the horizontal electric field at a $30 \mathrm{~km}$ altitude caused by a two-dimensionally convergent electric field in the ionosphere is almost the same as that in the ionosphere, and the ionospheric contribution to the vertical electric field on the ground could amount to 20 times that in the ionosphere, when the ionospheric electric field exists in an ionospheric belt with a width of $200 \mathrm{~km}$. Thus, the ionospheric contribution to the field intensity is expected to be $1 \mathrm{~V} / \mathrm{m}$ on the ground when it is $50 \mathrm{mV} / \mathrm{m}$ in the ionosphere.

3) The ionospheric contributions to electric field intensity and its fluctuation below the ionosphere highly depend on the horizontal size of the ionospheric 


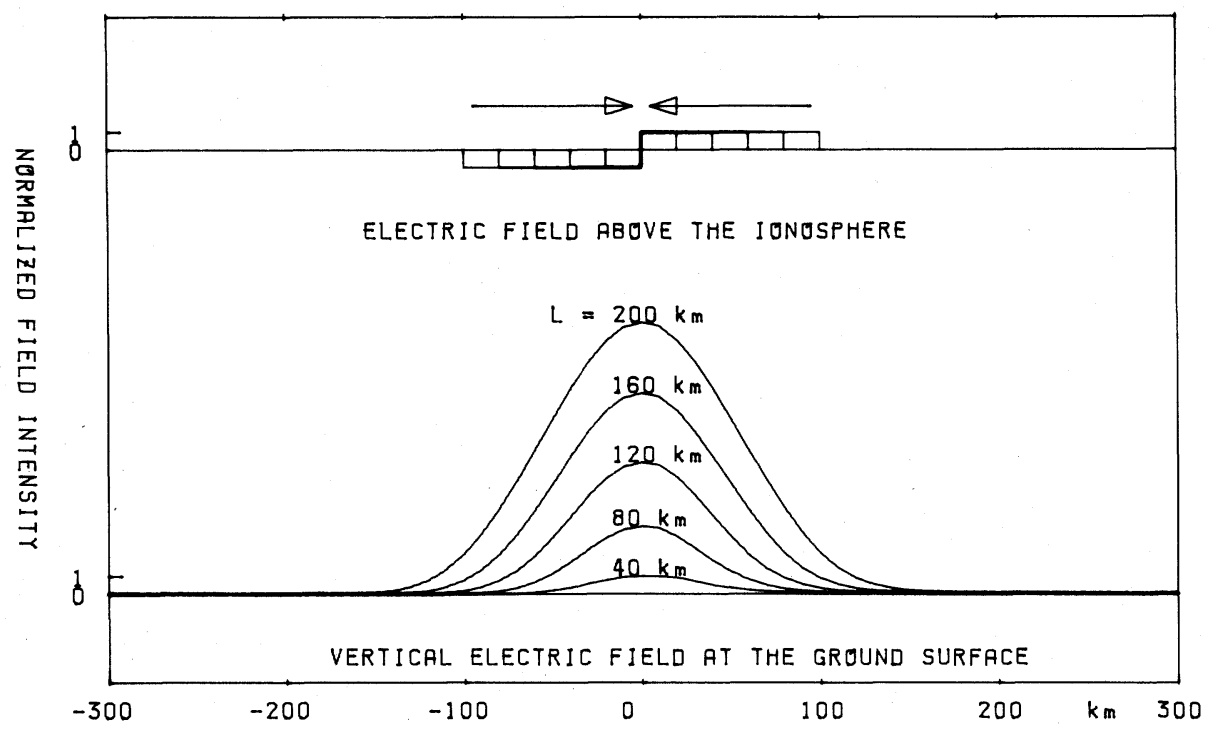

(b)

Fig. 7. (a) Electric field distribution below the ionosphere when a two-dimensionally convergent electric field exists in the north-south direction in and above the ionosphere within an east-west elongated belt, $200 \mathrm{~km}$ in width. The upward electric field on the ground under the convergence region in the ionosphere electric field is found to be about 20 times the horizontal component in the ionosphere. (b) Intensity distribution of the vertical electric field on the ground, the characteristic scale size of the ionospheric electric field distribution, $L$, as parameters. The ionospheric electric field is two-dimensionally convergent in the same way as in Fig. 7(a). The ground electric field is again found to be highly dependent on the characteristic scale size of the ionospheric electric field.

electric field. Both could amount to a measurable range when the size of the ionospheric electric field or auroral activity is larger than some tens to a hundred $\mathrm{km}$.

4) Even when the intensity of the ionospheric contribution amounts to the measurable range, separation of the ionospheric contribution from the background would require some techniques, such as multiple correlations between electric field fluctuations and luminosities of auroral patches, and spatial correlations of electric field variations among network stations.

One of the most outstanding features of the electric field below the ionosphere is that the electric field with a small horizontal extent can penetrate down to the lower altitudes. This is due to the steep decrease in electric conductivity below the ionosphere. An important nature of the electric field is that the electric field on the ground is an image with a higher spatial resolution of the ionospheric electric field in contrast to the much more "out of focus" image of the magnetic 
field produced in the ionosphere and observed on the ground. Although we did not show the distribution pattern of the vertial electric field on the ground, it is almost the same as that at the balloon altitude in Fig. 4(a) except horizontal components. Very little horizontal components appear on the ground because of the high ground conductivity.

The ground observation of the ionospheric electric field, therefore, requires fine grids of stations situated with a distance of $50 \mathrm{~km}$ or smaller, for comparing the spatial distributions of observed electric fields with calculations, and for taking reliable spatial correlations between stations. At the same time, the whole extent of the station network is desirable to cover the whole area of auroral activity and the possible extent of the ionospheric electric field in order to determine the distribution pattern. One might wonder if $50 \mathrm{~km}$ grid is fine enough to detect the contributions of much smaller auroral patches since it is well known that most pulsating auroral patches are usually smaller than $50 \mathrm{~km}$ across. However, as seen in Fig. 6, the contribution remarkably decreases as the size is reduced, and hence it would become undetectable by any means, even if the multiple correlation method is applied. The $50 \mathrm{~km}$, or a little smaller, grids proposed here may be necessary and sufficient to obtain significant results of the ground measurements of the ionospheric electric fields.

The enhancement of the vertical electric field near the earth's surface and its steep decrease upward are other important natures of the electric field in the atmosphere. This is due to a space charge distribution in the atmosphere with non-uniform conductivity and at the earth's surface. The space charge effect is notable at and in the vicinity of the earth's surface, due to the stepwise change in conductivity at the surface. This effect steeply decreases with increase in altitude, and subsequently does so also the vertical electric field in the vicinity of the earth's surface.

The derivation of the electric field below the ionosphere in this study is based on a thin sheet model of the ionosphere. The gradual change in the electric field from a two-dimensional form above to a three-dimensional form below the ionospheric boundary, as well as the continuous change from anisotropic electric conductivities above to isotropic conductivity below the boundary, is not taken into consideration in this calculation. The penetration of the electric field from above to below the ionospheric boundary in a rigorous treatment, however, is expected not to be far from this approximation as far as the electric field on the ground, remote from the ionosphere, is concerned.

\section{REFERENCES}

MiUra, A., S. OHtsuka, and T. TAmao, Coupling instability of the shear Alfven wave in the magnetosphere with the ionospheric ion drift wave: 2. Numerical analysis, J. Geophys. Res., 87, 843-851, 1982.

MOZER, F. S. and P. BRUSTON, Electric field measurements in the auroral ionosphere, J. Geophys. Res., 72, 1109-1114, 1967.

Mozer, F. S. and R. SERLIN, Magnetospheric field measurements with balloons, J. Geophys. Res., 
74, 4739-4754, 1969.

OGuti, T., TV observations of auroral arcs, in Physics of Auroral Arc Formation, Geophys. Monograph Ser., Vol. 25, pp. 31-41, A.G.U., 1981.

OGUTI, T. and K. HAYASHI, Multiple correlation between auroral and magnetic pulsations: 2-Determination of electric currents and electric fields around a pulsating auroral patch, J. Geophys. Res., 89, 7467-7481, 1984.

OGUti, T., J. H. MEEK, and K. HAYASHI, Multiple correlation between auroral and magnetic pulsations, J. Geophys. Res., 89, 2295-2303, 1984.

PARK, C. G., Downward mapping of high latitude ionospheric electric fields to the ground, $J$. Geophys. Res., 81, 168-174, 1976.

StUBBE, P. and H. KOPKA, Modulation of the polar electrojet by powerful HF waves, J. Geophys. Res., 82, 2319-2325, 1977. 